\title{
Polymyxin Resistance Among XDR ST1 Carbapenem-Resistant Acinetobacter baumannii Clone Expanding in a Teaching Hospital
}

\section{OPEN ACCESS}

Edited by:

Spyros Pournaras,

National and Kapodistrian University

of Athens, Greece

Reviewed by:

Carlos Henrique Camargo,

Adolfo Lutz Institute, Brazil

Eliana Pia Esposito,

University of Naples Federico II, Italy

*Correspondence:

Ilana Lopes Baratella da Cunha

Camargo

ilanacamargo@ifsc.usp.br

tThese authors have contributed equally to this work

Specialty section: This article was submitted to Antimicrobials, Resistance and Chemotherapy,

a section of the journal

Frontiers in Microbiology

Received: 29 October 2020 Accepted: 05 March 2021 Published: 26 March 2021

Citation:

Carrasco LDM, Dabul ANG,

Boralli CMS, Righetto GM,

Carvalho IS, Dornelas JV, Martins da Mata CPS, Araújo CA,

Leite EMM, Lincopan N and Camargo ILBC (2021) Polymyxin

Resistance Among XDR ST1

Carbapenem-Resistant Acinetobacter baumannii Clone Expanding in a

Teaching Hospital.

Front. Microbiol. 12:622704. doi: 10.3389/fmicb.2021.622704
Letícia Dias de Melo Carrasco'1t, Andrei Nicoli Gebieluca Dabul'1t, Camila Maria dos Santos Boralli', Gabriela Marinho Righetto', lago Silva e Carvalho', Janaína Valerini Dornelas ${ }^{1}$, Camila Pacheco Silveira Martins da Mata ${ }^{2}$, César Augusto de Araújoº ${ }^{2}$ Edna Mariléa Meireles Leite ${ }^{2}$, Nilton Lincopan $^{3}$ and Ilana Lopes Baratella da Cunha Camargo ${ }^{\text {* }}$

1 Laboratory of Molecular Epidemiology and Microbiology, Department of Physics and Interdisciplinary Science, São Carlos Institute of Physics, University of São Paulo, São Paulo, Brazil, ${ }^{2}$ Hospital Risoleta Tolentino Neves, Belo Horizonte, Brazil,

${ }^{3}$ Department of Microbiology, Institute of Biomedical Sciences, University of São Paulo, São Paulo, Brazil

Acinetobacter baumannii is an opportunistic pathogen primarily associated with multidrug-resistant nosocomial infections, for which polymyxins are the last-resort antibiotics. This study investigated carbapenem-resistant $A$. baumannii strains exhibiting an extensively drug-resistant (XDR) phenotype, including four isolates considered locally pan drug-resistant ( $\mathrm{PDR}$ ), isolated from inpatients during an outbreak at a teaching hospital in Brazil. Apal DNA macrorestriction followed by PFGE clustered the strains in three pulsotypes, named $\mathrm{A}$ to $\mathrm{C}$, among carbapenem-resistant $A$. baumannii strains. Pulsotypes $A$ and $B$ clustered six polymyxin-resistant $A$. baumannii strains. MLST analysis of representative strains of pulsotypes $A, B$, and $C$ showed that they belong, respectively, to sequence types ST1 (clonal complex, CC1), ST79 (CC79), and ST903. Genomic analysis of international clones ST1 and ST79 representative strains predicted a wide resistome for $\beta$-lactams, aminoglycosides, fluoroquinolones, and trimethoprim-sulfamethoxazole, with blaOXA-23 and blaOXA-72 genes encoding carbapenem resistance. Amino acid substitutions in PmrB (Thr232lle or Pro170Leu) and PmrC (Arg125His) were responsible for polymyxin resistance. Although colistin MICs were all high ( $\mathrm{MIC} \geq 128 \mathrm{mg} / \mathrm{L}$ ), polymyxin B MICs varied; strains with Pro170Leu substitution in PmrB had MICs > $128 \mathrm{mg} / \mathrm{L}$, while those with Thr232lle had lower MICs (16-64 mg/L), irrespective of the clone. Although the first identified polymyxin-resistant A. baumannii strain belonged to ST79, the ST1 strains were endemic and caused the outbreak most likely due to polymyxin B use. The genome comparison of two ST1 strains from the same patient, but one susceptible and the other resistant to polymyxin, revealed mutations in 28 ORFs in addition to pmrBC. The ORF codifying an acyl-CoA dehydrogenase has gained attention due to its fatty acid breakdown and membrane fluidity involvement. However, the role of these mutations in the polymyxin resistance 
mechanism remains unknown. To prevent the dissemination of XDR bacteria, the hospital infection control committee implemented the patient bathing practice with a $2 \%$ chlorhexidine solution, a higher concentration than all $A$. baumannii chlorhexidine MICs. In conclusion, we showed the emergence of polymyxin resistance due to mutations in the chromosome of the carbapenem-resistant A. baumannii ST1, a high-risk global clone spreading in this hospital.

Keywords: carbapenem-resistant Acinetobacter baumannii, polymyxin resistance, extensively drug-resistant, pan drug-resistant, A. baumannii ST1, A. baumannii ST79, PmrC, PmrB

\section{INTRODUCTION}

Acinetobacter baumannii is an opportunistic pathogen often associated with hospital-acquired infections worldwide, especially in intensive care units (ICUs) (Gundi et al., 2009; Zhang et al., 2013; Lin and Lan, 2014). A. baumannii has high rates of resistance to multiple antimicrobials due to its propensity to rapidly acquire resistance genes or even due to intrinsic resistance mechanisms typical to the genus (Peleg et al., 2008; Durante-Mangoni and Zarrilli, 2011). Furthermore, A. baumannii growth structured as biofilms on medical devices and mucous surfaces difficult their elimination, contributing to cause persistent and recurrent infections (Rodríguez-Baño et al., 2008). Epidemiologically, A. baumannii is one of the most severe ESKAPE pathogens (Enterococcus faecium, Staphylococcus aureus, Klebsiella pneumoniae, A. baumannii, Pseudomonas aeruginosa, and Enterobacter spp.) (Boucher et al., 2009). In addition, carbapenem-resistant $A$. baumannii is on the top of the World Health Organization (WHO) list of critical priorities for developing new antimicrobials (Tacconelli et al., 2018).

Polymyxins (colistin and polymyxin B) are antibiotics from the 1960 s reintroduced in the 2000s in an attempt to treat infections caused by multidrug-resistant (MDR) gramnegative bacteria, particularly those resistant to carbapenems, as last-resort antibiotics (Karaiskos and Giamarellou, 2014). Combination therapy, such as polymyxins/aminoglycosides or meropenem or tigecycline, is considered when A. baumannii is resistant to carbapenem but susceptible to these antibiotics (Satlin et al., 2020). However, the use of tigecycline is not recommended or at least controversial ( $\mathrm{Ni}$ et al., 2016). On the other hand, the meropenem/colistin combination improves survival in critically ill patients infected with carbapenem-resistant A. baumannii (Park et al., 2019). Since their reintroduction into clinical practice, polymyxins still display good activity against non-fermentative pathogens (Dias et al., 2016); however, resistance has increased in recent years (Karaiskos and Giamarellou, 2014).

At first, the polymyxin resistance mechanisms were only chromosomally related and, therefore, more difficult to disseminate (Cai et al., 2012). Later, plasmid-mediated genes conferring resistance to polymyxins were described, the so-called mor genes. Initially, researchers thought that the $\mathrm{mor}$ genes were only among Enterobacterales (Fernandes et al., 2016; Liu et al., 2016, 2017; Xavier et al., 2016; Kluytmans, 2017; Rau et al., 2018). However, the description of the mor gene in Acinetobacter spp. revealed that this gene represents a real threat for rapid dissemination of resistance to the last resort therapeutic option available to face infections caused by gram-negative MDR bacteria (Hameed et al., 2019; Ma et al., 2019; Martins-Sorenson et al., 2020).

In Brazil, Acinetobacter spp. have exhibited elevated carbapenem resistance rates, remaining susceptible to polymyxins (Gales et al., 2012; Dias et al., 2016; Leite et al., 2016; Rossi et al., 2017). In the present study, we aimed to perform a phenotypic and molecular comparison of carbapenem-resistant A. baumannii strains exhibiting extensively drug-resistant (XDR) profile (Magiorakos et al., 2012) isolated from inpatients at a teaching hospital to determine whether there was an outbreak of a specific clone and to investigate the molecular mechanisms underlying the polymyxin resistance development.

\section{MATERIALS AND METHODS}

\section{Bacterial Isolates and Antimicrobial Susceptibility Testing}

We studied sixteen Acinetobacter baumannii strains recovered from 14 patients admitted to a teaching hospital in Minas Gerais state, in Southeastern Brazil (Figure 1). This was an observational and non-interventionist study using bacterial samples only, which did not require consent procedures, and was approved by the collegiate of the teaching, research and extension unitNEPE/HRTN ethical committee (NEPE 26/2017). Serving an average of 120 patients per day in the emergency and maternity wards, this teaching hospital has 345 beds distributed in the following wards: 111 belong to the emergency, 96 to the medical clinic, 72 to surgery, 35 to intensive care unit, and 31 to maternity.

The isolates were stored in tryptic soy broth containing $40 \%$ glycerol at $-80^{\circ} \mathrm{C}$, and cultured on Brain Heart Infusion (BHI) agar plates at $37{ }^{\circ} \mathrm{C}$ for $24 \mathrm{~h}$ before performing the tests. Bacterial identification and antimicrobial susceptibility tests were determined using the Vitek 2 Compact (bioMérieux, France). Additionally, susceptibility to amikacin, gentamicin, imipenem, meropenem, ciprofloxacin, piperacillin-tazobactam, ampicillin-sulbactam, cefotaxime, ceftazidime, trimethoprimsulfamethoxazole, and tetracycline was determined by the disk diffusion method, according to the Clinical and Laboratory Standards Institute guidelines (Clinical and Laboratory Standards Institute, 2018). Minimal inhibitory concentrations (MICs) for colistin and polymyxin B (purchased from GoldBio, United States, and Sigma-Aldrich, United States, respectively) 

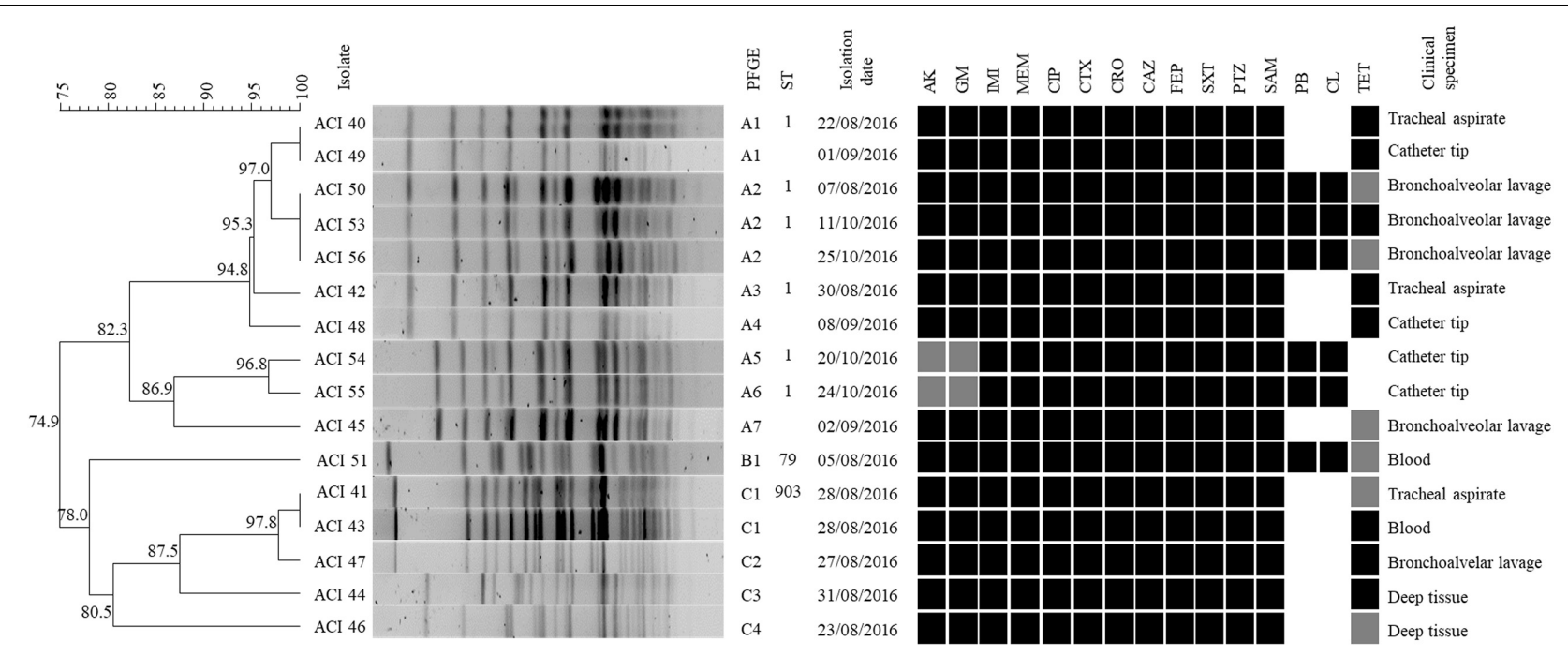

FIGURE 1 | Genotypic and phenotypic characteristics of the A. baumannii isolates. From left to right, the figure shows the PFGE dendrogram with the percentage of genetic similarity, the A. baumannii isolates, PFGE subtype definition, sequence type (ST) according to Pasteur MLST scheme, isolation date, susceptibility profile, and clinical specimen. The following antimicrobials were tested: AK, Amikacin; CN, Gentamicin; IMI, Imipenem; MEM, Meropenem; CIP, Ciprofloxacin; CTX, Cefotaxime; CRO, Ceftriaxone; CAZ, Ceftazidime; FEP, Cefepime; SXT, Trimethoprim-sulfamethoxazole; PTZ, Piperacillin-tazobactam; SAM, Ampicillin-sulbactam; PB, Polymyxin B; CL, Colistin; TET, Tetracycline. Black, gray, and white squares represent resistant, intermediate, and susceptible, respectively.

and tigecycline (Wyeth, United States) were determined in duplicate using the broth microdilution method (Eucast, 2017; Clinical and Laboratory Standards Institute, 2018). It is important to note that the European Committee on Antimicrobial Susceptibility Testing (EUCAST) indicates that there is insufficient evidence that Acinetobacter species are a good target for therapy with tigecycline and that we should report the MIC value without an interpretation.

Multidrug-resistant (MDR) and extensively drug-resistant (XDR) profiles were defined using previously established criteria (Magiorakos et al., 2012). Because not all antibiotics suggested in the criteria mentioned above were available for use in this hospital, the strains were considered locally pan drug-resistant ( $\left.{ }_{\mathrm{L}} \mathrm{PDR}\right)$ when resistant to all antibiotics tested in this study.

In addition, we determined the MIC to chlorhexidine gluconate by broth microdilution method using cationadjusted Mueller-Hinton broth, since patient bathing with $2 \%$ chlorhexidine is a protocol to control the spread of MDR pathogens in this hospital. For this, we diluted $20 \%(\mathrm{w} / \mathrm{v}$ aqueous solution) chlorhexidine digluconate (Neon Comercial Reagentes Analíticos, Brazil) to obtain final concentrations ranging from 256 to $1 \mathrm{mg} / \mathrm{L}$.

\section{Identification of $A$. baumannii Species by PCR}

For PCR amplification, the clinical strains genomic DNA was mechanically extracted, following an adapted methodology previously described (Palazzo et al., 2007). A. baumannii species was confirmed by detection of the bla $O X A-51-l i k e$ gene. Amplification of this gene was performed with primers previously described (Sohrabi et al., 2012), in a final volume of $25 \mu \mathrm{L}$, containing $100 \mathrm{ng}$ of genomic DNA, $0.2 \mu \mathrm{M}$ of each primer, $2.5 \mathrm{mM}$ of each dNTP, $2.0 \mathrm{mM}$ of $\mathrm{MgCl}_{2}$ and $1 \mathrm{U}$ Taq DNA Polymerase (Cellco, Brazil), under the following cycling conditions: initial denaturation $\left(95^{\circ} \mathrm{C} / 1 \mathrm{~min}\right), 35$ cycles of denaturation $\left(95^{\circ} \mathrm{C} / 30 \mathrm{~s}\right)$, annealing $\left(57.5^{\circ} \mathrm{C} / 30 \mathrm{~s}\right)$ and extension $\left(72^{\circ} \mathrm{C} / 1 \mathrm{~min}\right)$, and a final extension $\left(72^{\circ} \mathrm{C} / 2 \mathrm{~min}\right)$ in a thermal cycler (C1000, Bio-Rad Laboratories Inc., United States). The PCR products were separated by $1.5 \%$ agarose gel electrophoresis, stained with $0.01 \%$ SYBR Safe (Invitrogen, Life Technologies, United States), and visualized using the ChemiDoc XRS imaging system (Bio-Rad Laboratories Inc., United States).

\section{Pulsed-Field Gel Electrophoresis}

The strains genetic relatedness was observed by DNA macrorestriction with $30 \mathrm{U}$ of ApaI enzyme (New England BioLabs Inc., United States) followed by pulsed-field gel electrophoresis (PFGE), as previously described (Durmaz et al., 2009). The electrophoresis was run with $0.5 \mathrm{X}$ TBE buffer at $14^{\circ} \mathrm{C}$, with $6 \mathrm{~V} / \mathrm{cm}^{2}$, for $20 \mathrm{~h}$ and linear ramping, with initial and final switch times of 5 and $30 \mathrm{~s}$, respectively, using a CHEF Mapper system (CHEF Mapper XA Pulsed Field Electrophoresis System-Bio-Rad Laboratories Inc., United States). After electrophoresis, the gel was stained with $0.01 \%$ SYBRSafe (Invitrogen, Life Technologies, United States) in 0.5x TBE buffer for $1 \mathrm{~h}$, followed by visualization using the ChemiDoc XRS (Bio-Rad Laboratories Inc., United States).

We compared the DNA band profiles using the Bionumerics software (version 7.6, Applied-Maths, Belgium) with 0.5\% optimization and $1.25 \%$ tolerance parameters, with cluster analysis done by the unweighted pair group method using the arithmetic average (UPGMA). Isolates with 100\% similarity by PFGE were considered indistinguishable and clustered in the same pulsotype and subtype; isolates with similarity $80 \%$ 
were considered closely related and clustered in the same pulsotype, but different subtypes; isolates with similarity $<80 \%$ were considered possibly related and are classified as belonging to different pulsotypes.

\section{Whole-Genome Sequencing, MLST, and Resistome Analysis}

For whole-genome sequencing, the genomic DNA of ACI40, ACI42, ACI50, ACI51, ACI53, ACI54, and ACI55 strains was extracted according to the manufacturer instructions using the DNeasy blood and tissue kit (Qiagen $\mathrm{GmbH}$, Germany). Libraries were prepared from $1 \mathrm{ng}$ total DNA using a Nextera XT Sample Preparation Kit (Illumina, United States), with modifications for 2 x 250 bp paired-end sequencing. Samples were sequenced using MiSeq or NextSeq platforms (Illumina, United States). CLC Genomics Workbench v.10.1.1 (QIAGEN, Denmark) was used for de novo genome assembly. Contigs were annotated through the NCBI Prokaryotic Genome Annotation Pipeline, and genomes were deposited at DDBJ/EMBL/GenBank. For A. baumannii strains ACI40, ACI42, ACI50, ACI53, ACI54, ACI55, and ACI51, sequence types (STs) were determined using the MLST 1.8 online tool from the Center for Genomic Epidemiology ${ }^{1}$.

For the ACI41 strain, the DNA was extracted and submitted to PCR amplification using MLST primers indicated by the Pasteur scheme ${ }^{2}$. PCR products were sequenced using the Sanger method (Diancourt et al., 2005). Chromatograms were checked using Vector NTi (Invitrogen, United States) and submitted to the Pasteur database in order to obtain the sequence type (ST), as determined by the combination of alleles identified ${ }^{3}$. Resistome of strains ACI40, ACI42, ACI50, ACI53, ACI54, ACI55, and ACI51 was predicted by using ResFinder $3.2^{4}$ and the Comprehensive Antibiotic Resistance Database (CARD) ${ }^{5}$. The presence of mutations associated with polymyxin resistance was investigated by the alignment of eptA, pmrCAB, adeRS, lpsB, and $l p x A C D$ genes (Hood et al., 2013; Lesho et al., 2013; Moffatt et al., 2019; Gerson et al., 2020; Yilmaz et al., 2020) from polymyxin-resistant (ACI50, ACI51, ACI53, ACI54, ACI55) and polymyxin-susceptible isolates (ACI40 and ACI42), whereas the genome of A. baumannii AB030 (NZ_CP009257.1) was used as colistin-susceptible reference strain (Loewen et al., 2014). For comparison, the $p m r B$ gene of $A$. baumannii ACI56 was amplified with primers and conditions already described (Beceiro et al., 2011) in a thermal cycler (C1000, Bio-Rad Laboratories Inc., United States). The PCR product was separated by $1.5 \%$ agarose gel electrophoresis, stained with 0.01\% SYBR Safe (Invitrogen, Life Technologies, United States), and visualized in the ChemiDoc XRS image system (Bio-Rad Laboratories Inc., United States). The fragment was purified by the PCR purification kit (Jena Biosciences, Germany) and sequenced by the Sanger method at the Laboratory of Biophysics

\footnotetext{
${ }^{1}$ https://cge.cbs.dtu.dk/services/MLST

${ }^{2}$ https://pubmlst.org/bigsdb?db=pubmlst_abaumannii_pasteur_seqdef

${ }^{3}$ https://pubmlst.org/organisms/acinetobacter-baumannii

${ }^{4}$ https://cge.cbs.dtu.dk/services/ResFinder/

${ }^{5}$ https://card.mcmaster.ca/home
}

Sergio Mascarenhas at the IFSC-USP. Chromatograms were checked using Vector NTi (Invitrogen, United States) and compared to the other isolates genomes' genes. The genome sequences were submitted to GenBank, and accession numbers are as follows: WJWR00000000 (ACI40), WJWS00000000 (ACI42), PNFN00000000 (ACI50), PNJH00000000 (ACI51), WJWT00000000 (ACI53), PNJI00000000 (ACI54), and PNFO00000000 (ACI55).

\section{Determination of the Doubling Time}

The bacteria ACI40 and ACI50 were incubated in Mueller Hinton Cation Adjusted broth at $37^{\circ} \mathrm{C}$ for $18 \mathrm{~h}$. The suspension was adjusted to $\mathrm{OD}_{600}$ of $0.08-1.0$, and $0.2 \mathrm{ml}$ were transferred to each well in the microplate. The growth was measured by absorbance $\left(\mathrm{OD}_{600}\right)$ using the spectrophotometer SpectraMax M5 (Molecular Devices). For growth curve and doubling time determinations, the OD vs. time was plotted for each strain in the exponential growth phase. The doubling times were then calculated as follows: $[(\mathrm{t} 2-\mathrm{t} 1) \times \log 2] /\left(\log \mathrm{OD}_{600}\right.$ at $\mathrm{t} 2-\log$ $\mathrm{OD}_{600}$ at $\mathrm{t} 1$ ), where $\mathrm{t} 1$ is sampling time 1 and $\mathrm{t} 2$ is sampling time 2. Two independent experiments were carried out in 6 replicates. The doubling time data were compared by ANOVA single factor.

\section{Biofilm Formation}

The quantitative biofilm formation (biofilm mass) of A. baumannnii clinical strains was evaluated according to Qin et al. (2014) with modifications. Briefly, an isolated colony from a fresh culture was inoculated in $35 \mathrm{~mL}$ of BHI broth with $0.75 \%$ glucose and incubated $\left(37^{\circ} \mathrm{C}\right.$ for $\left.24 \mathrm{~h}\right)$. After that, this culture was centrifuged $\left(4,100 \mathrm{rpm} / 4^{\circ} \mathrm{C} / 10 \mathrm{~min}\right)$, the pellet was resuspended in $0.5 \mathrm{~mL}$ of $\mathrm{BHI}$ broth $+0.75 \%$ glucose, and $0.05 \mathrm{ml}$ of this suspension was added into $0.45 \mathrm{ml}$ of phosphatebuffered saline $(\mathrm{PBS})$, to reach the $\mathrm{OD}_{600}=1.0$. This adjusted suspension was then diluted (1:40) in BHI broth $+0.75 \%$ glucose, and $0.2 \mathrm{ml}$ was added to the wells of a flat bottom 96-wells microplate in triplicate. Staphylococcus epidermidis ATCC 35984 (good biofilm-forming strain) and Staphylococcus epidermidis ATCC 12228 (non-biofilm-forming strain) were used as positive and negative controls, respectively. Fresh BHI broth $+0.75 \%$ glucose was considered as blank. The microplate was incubated at $37^{\circ} \mathrm{C}$ during $24 \mathrm{~h}$, the culture medium was removed, the wells were washed thrice with PBS, and let dry at room temperature. Then, $0.2 \mathrm{ml}$ of crystal violet $(0.2 \%)$ was added to each well to stain the biofilms for $15 \mathrm{~min}$, and the wells were washed thrice with PBS. After that, $0.2 \mathrm{ml}$ of ethanol:acetone solution (80:20) was added, and the microplates were shaken for $1 \mathrm{~min}$. After homogenization, $0.04 \mathrm{ml}$ of each well's content was added to $0.16 \mathrm{ml}$ of ethanol:acetone solution in a new microplate, and the absorbance was measured at $\mathrm{OD}_{595}$ in the microplate reader SpectraMax M5 (Molecular Devices, United States). The negative and positive controls (S. epidermidis ATCC 12228 and S. epidermidis ATCC 35984) were compared using the two-tailed $t$-test, for which the result was considered acceptable when the $p$ value was lower than 0.05 . To determine if the isolates produced a significant amount of biofilm, they were compared by the twotailed $t$-test with the negative control S. epidermidis ATCC 12228, being considered statistically different from the negative control, 
and therefore biofilm formers, when the $p$-value was lower than 0.05. For these cases, the percentage of biofilm formation in comparison with the negative control was calculated.

\section{RESULTS}

\section{Extensively Drug-Resistant $A$. baumannii ST1 Spread Among Patients}

All isolates were confirmed as A. baumannii by the presence of the species-specific bla $a_{O X A-51-l i k e}$ gene. The MICs of colistin, polymyxin B, and tigecycline are in Table 1. Six out of 16 A. baumannii isolates were resistant to both polymyxins representing a $37.5 \%$ incidence of polymyxin resistance in the study period. Of these polymyxin-resistant strains, only one was susceptible to tetracycline, and another was susceptible to tetracycline and tigecycline. Susceptibility to tigecycline was determined according to PK/PD breakpoints for non-species related (Eucast, 2017). Considering the parameters of Magiorakos et al. (2012), all the analyzed strains are XDR. Four of them were non-susceptible to all the 16 different antimicrobials tested, belonging to nine different antimicrobial categories, and were classified as ${ }_{\mathrm{L}}$ PDR due to the hospital antimicrobial availability. The chlorhexidine MIC of all isolates ranged from 16 to $32 \mathrm{mg} / \mathrm{L}$ (Table 1).

Figure 1 shows the genetic similarity between the 16 A. baumannii strains by PFGE. Pulsotype A is the most prevalent among the A. baumannii strains isolated in this hospital (62.5\%), followed by pulsotypes $\mathrm{C}$ and B (31.2 and 6.2\%, respectively).

The strains clustered in the pulsotype A include seven different subtypes (A1-A7), 40\% $(n=4)$ isolated from bronchoalveolar lavage, $40 \%(n=4)$ from catheter tips, and $20 \%(n=2)$ from tracheal aspirates. There are some indistinguishable strains

TABLE 1 | MIC values of antibiotics and chlorhexidine for A. baumannii isolates.

\begin{tabular}{|c|c|c|c|c|}
\hline \multirow[t]{2}{*}{ Isolates } & \multicolumn{4}{|c|}{$\operatorname{MIC}(\mathrm{mg} / \mathrm{L})_{a}$} \\
\hline & Colistin & Polymyxin B & Tigecycline & Chlorhexidine \\
\hline $\mathrm{ACl} 40$ & 0.5 & 0.25 & 2 & 32 \\
\hline $\mathrm{ACl} 41$ & 0.5 & 0.25 & 2 & 32 \\
\hline $\mathrm{ACl} 42$ & 0.25 & 0.25 & 2 & 32 \\
\hline $\mathrm{ACl} 43$ & 0.25 & 0.25 & 2 & 32 \\
\hline $\mathrm{ACl} 44$ & 0.5 & 0.25 & 1 & 32 \\
\hline $\mathrm{ACl} 45$ & 0.25 & 0.25 & 1 & 16 \\
\hline $\mathrm{ACl} 46$ & 0.5 & 0.25 & 2 & 32 \\
\hline $\mathrm{ACl} 47$ & 0.5 & 0.25 & 2 & 32 \\
\hline $\mathrm{ACl} 48$ & 0.5 & 0.25 & 2 & 32 \\
\hline $\mathrm{ACl} 49$ & 0.5 & 1 & 2 & 32 \\
\hline $\mathrm{ACl} 50$ & $>128$ & 128 & 2 & 16 \\
\hline $\mathrm{ACl} 51$ & $>128$ & 64 & 2 & 16 \\
\hline $\mathrm{ACl} 53$ & $>128$ & $>128$ & 8 & 32 \\
\hline $\mathrm{ACl} 54$ & $>128$ & 64 & 1 & 16 \\
\hline ACl55 & 128 & 16 & 0.25 & 16 \\
\hline ACl56 & $>128$ & $>128$ & 2 & 16 \\
\hline
\end{tabular}

${ }^{a}$ Resistance in bold. clustered in the pulsotype A: two strains clustered in the subtype A1 (ACI40 and ACI49), and three strains clustered in the subtype A2 (ACI50, ACI53, and ACI56). The subtype A1 strains were isolated from different dates and clinical specimens (Figure 1), patients and units (data not shown), but both of them showed the same antimicrobial susceptibility profile (Figure 1). The subtype A2 strains were non-susceptible to all the antimicrobials evaluated in this study (Figure 1). Furthermore, they were all isolated from bronchoalveolar lavage (Figure 1) from different patients in the same intensive care unit (ICU) (data not shown), but at different dates (Figure 1).

The ACI51 was the first strain temporarily isolated during the study period and the only strain belonging to the pulsotype B. It was isolated from blood and showed resistance to the polymyxins (Figure 1), so it was the first polymyxin-resistant A. baumannii strain detected in this hospital. The other five polymyxin-resistant isolates (ACI50, ACI53, ACI54, ACI55, and ACI56) were clustered in the pulsotype A (Figure 1).

Pulsotype $\mathrm{C}$ consists of four subtypes (C1-C4), being all of them susceptible to polymyxins. They were all isolated within eight days, except for two indistinguishable strains (ACI41 and ACI43) clustered in the subtype C1 that were isolated on the same date in different clinical specimens (Figure 1) from the same patient admitted to the ICU (data not shown). These data suggest this patient had a severe disseminated infection caused by one polymyxin susceptible A. baumannii clone.

Of the representative isolates with their ST determined, those from pulsotype A belong to ST1 and ST405, and the pulsotype B isolate belongs to ST79 and ST233 (Pasteur and Oxford MLST schemes, respectively). One representative of pulsotype C1 belongs to the ST903 (Pasteur MLST scheme) (Figure 1).

\section{Molecular Basis for the Resistance Phenotype of the A. baumannii Strains and Fitness Comparison}

The genomes coverages varied from $128 \mathrm{x}$ to $251 \mathrm{x}$, and the details of contigs and GenBank accession numbers are in Supplementary Table S1. The acquired resistance genes detected in the draft genomes by Resfinder are in Table 2.

In addition to the $a a c\left(6^{\prime}\right) I b-c r$ gene conferring quinolone resistance, CARD showed that all isolates have mutations leading to the Ser81Leu amino acid substitution in GyrA. The parC gene of the ST1 isolates also has mutations leading to Ser84Leu, Val104Ile, and Aps105Glu amino acid substitutions. ACI51 has only Val104Ile and Aps105Glu amino acid substitutions in ParC.

The tigecycline-resistance gene codifying TetX was absent in all isolates. AdeABC efflux pump and its two-component system AdeSR, both involved in multidrug resistance, including tigecycline (Ruzin et al., 2010), were present in all draft genomes. Only ACI54 and ACI55 presented the nucleotide substitution G40A in the adeS gene leading to Ala14Thr substitution in AdeS.

Specifically, all variants already known of the $\mathrm{mor}$ plasmidmediated gene and the eptA gene were absent in the genomes analyzed regarding polymyxin resistance.

We extracted the genes that were already reported by others as involved in polymyxins resistance $l p x A C D$ and $p m r C A B$ from 
TABLE 2 | Resistome and mutations related to polymyxin resistance identified in the sequenced genomes of $A$. baumannii strains.

\begin{tabular}{|c|c|c|c|c|c|c|c|}
\hline \multirow[t]{2}{*}{ Characteristics } & \multicolumn{7}{|c|}{ Acinetobacter baumannii strains } \\
\hline & $\mathrm{ACl} 40$ & ACl42 & ACI50 & $\mathrm{ACI} 53$ & ACI54 & ACI55 & ACI51 \\
\hline Polymyxin susceptibility & $S$ & $S$ & $\mathrm{R}$ & $\mathrm{R}$ & $\mathrm{R}$ & $\mathrm{R}$ & $\mathrm{R}$ \\
\hline MLST (ST) & ST1 & ST1 & ST1 & ST1 & ST1 & ST1 & ST79 \\
\hline \multicolumn{8}{|l|}{ Resistome } \\
\hline Aminoglycosides & $\begin{array}{c}\operatorname{aac}\left(6^{\prime}\right) / b 3, \\
a p h\left(3^{\prime \prime}\right)-l b, \\
a p h\left(3^{\prime}\right)-I I, \text { aph(6)-Id }\end{array}$ & $\begin{array}{c}\operatorname{aac}\left(6^{\prime}\right) \text { Ib3, } \\
\text { aph }\left(3^{\prime \prime}\right)-l, a p h\left(3^{\prime}\right)-I l \\
\text { aph }(6)-l d\end{array}$ & 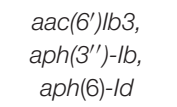 & $\begin{array}{c}\operatorname{aac}\left(6^{\prime}\right) \text { Ib3, } \\
\text { aph }\left(3^{\prime \prime}\right)-l, \text { aph }\left(3^{\prime}\right)-I l \\
\text { aph }(6)-l d\end{array}$ & $\begin{array}{l}\text { aac }\left(6^{\prime}\right) I b 3 \\
\text { aph(6)-ld }\end{array}$ & $\begin{array}{c}\text { aac }\left(6^{\prime}\right) / b 3 \\
\text { aph }\left(3^{\prime \prime}\right)-l b \\
\text { aph(6)-ld }\end{array}$ & $\begin{array}{c}\operatorname{aph}\left(3^{\prime \prime}\right)-I, \text { aph(6)-ld, } \\
\text { aadA1 }\end{array}$ \\
\hline$\beta$-lactams & $\begin{array}{l}\text { blaOXA-69, } \\
\text { bla } \\
\text { bla }_{A D C-23} \\
\text { bla }_{T E M-116}\end{array}$ & $\begin{array}{l}\text { blaOXA-69, } \\
\text { blaOXA-23, } \\
\text { blaADC-25, } \\
\text { blaTEM-116 }\end{array}$ & $\begin{array}{l}\text { blaOXA-69, } \\
\text { blaOXA-23, } \\
\text { blaADC-25 }\end{array}$ & $\begin{array}{l}\text { blaOXA-69, } \\
\text { blaOXA-23, } \\
\text { blaADC-25, } \\
\text { blaTEM-116 }\end{array}$ & $\begin{array}{l}\text { blaOXA-69, } \\
\text { blaOXA-23, } \\
\text { blaADC-25 }\end{array}$ & $\begin{array}{l}\text { blaOXA-69, } \\
\text { blaOXA-23, } \\
\text { blaADC-25 }\end{array}$ & $\begin{array}{l}\text { blaADC-25, } \\
\text { blaOXA-65, } \\
\text { blaOXA-72, } \\
\text { blaTEM-1A }\end{array}$ \\
\hline Fluoroquinolones & $\operatorname{aac}\left(6^{\prime}\right) / b-c r$ & $\operatorname{aac}\left(6^{\prime}\right) l b-c r$ & $\operatorname{aac}\left(6^{\prime}\right) / b-c r$ & $\operatorname{aac}\left(6^{\prime}\right) / b-c r$ & $\operatorname{aac}\left(6^{\prime}\right) / b-c r$ & $\operatorname{aac}\left(6^{\prime}\right) / b-c r$ & - \\
\hline Macrolides & & & $m p h(E), m s r(E)$ & $m p h(E), \operatorname{msr}(E)$ & $m p h(E), \operatorname{msr}(E)$ & $m p h(E), m s r(E)$ & $m p h(E), \operatorname{msr}(E)$ \\
\hline Phenicols & floR & floR & floR & floR & floR & floR & floR \\
\hline Sulfonamides & sul2 & sul2 & sul2 & sul2 & sul2 & sul2 & sul2 \\
\hline Trimethoprim & - & - & - & - & - & - & dfra1 \\
\hline Tetracyclines & - & - & - & tet(39) & - & - & \\
\hline pmrA mutations & - & - & - & - & - & - & G244A/Asp82Asn \\
\hline pmrB mutations & - & - & C509T/P170L & C509T/P170L & C695T/T232I & C695T/T232I & C695T/T232I \\
\hline pmrC mutations & - & - & G374A/R125H & G374A/R125H & G374A/R125H & G374A/R125H & G374A/R125H \\
\hline
\end{tabular}

draft genomes for a detailed analysis (Moffatt et al., 2010; Lesho et al., 2013; Gerson et al., 2020). We compared the genes of the polymyxin-resistant isolates (ACI50, ACI51, ACI53, ACI54, and ACI55) plus the susceptible ACI42 (ST1) to those of the susceptible isolate ACI40 (ST1). Because some amino acid substitutions are lineage-related, we also compared the proteins codified from ACI51 (ST79) genes to those of A. baumannii AB030 (ST79). The mutations detected are described in Table 2.

To investigate whether all strains with higher polymyxin $\mathrm{B}$ MIC (ACI50, ACI53, and ACI56) had the PmrB substitution Pro170Leu (Poirel et al., 2017), the $p m r B$ gene of ACI56, which did not have the draft genome sequenced, was PCR-amplified and sequenced by Sanger. As suspected, we found the same Pro170Leu substitution.

ACI40 (subtype A1) and ACI50 (subtype A2) were the only pulsotype A strains isolated from the same patient, from tracheal aspirate and bronchoalveolar lavage, respectively. They were closely related ( $97 \%$ similarity) by PFGE, but ACI40 was susceptible, and ACI50 was polymyxin-resistant. Comparing their genomes, we found 39 mutations leading to amino acid substitutions in 30 coding regions, including those already cited in $p m r B$ and $p m r C$ in ACI50 (Supplementary Table S2). We compared these coding regions to those in the other polymyxin-susceptible and resistant strains genomes sequenced in this study plus the polymyxin-susceptible ST79 AB030 strain genome. The coding regions were either absent in ST79 or were found only in ST1 polymyxin-resistant strains. Eight mutated coding regions, exclusive of ST1 polymyxin-resistant strains, codify two hypothetical proteins, an FMN-binding glutamate synthase family protein, Acyl-CoA dehydrogenase, a putative 2-aminoethyl phosphonate $\mathrm{ABC}$ transporter substratebinding protein, polysaccharide biosynthesis tyrosine autokinase, fumarylacetoacetate hydrolase, and an amidohydrolase family protein (Supplementary Table S2). In addition, we compared the growth curves of the two isolates (Supplementary Figure S1) and observed that the polymyxin-resistant ACI50 strain showed longer doubling time $(176 \pm 17 \mathrm{~min})$ compared with that of the susceptible strain ACI40 ( $148 \pm 1)$, with $p<0.005$ determined by the single-factor ANOVA.

\section{Biofilm Formation Is Not Enhanced Among the Polymyxin-Resistant Strains}

We wanted to investigate whether any correlation between polymyxin-resistance and biofilm formation existed. Figure 2 shows that all strains were able to form biofilms, which were statistically significant compared to the non-biofilm-former strain S. epidermidis ATCC 12228, or even compared to the negative control using Student's $t$-test, $p<0.05$. Thus, we considered all A. baumannii isolates as biofilm formers; The only isolate capable of forming biofilm comparable to the good biofilm-forming S. epidermidis ATCC 35984 strain was the polymyxin-susceptible ACI44 strain (Figure 2).

\section{DISCUSSION}

The isolation of gram-negative bacteria resistant to polymyxin at the Brazilian teaching hospital was unusual. Since the first polymyxin-resistant Acinetobacter baumannii complex isolate identification in that hospital, healthcare workers closely monitored and collected the subsequent Acinetobacter spp. isolates during the following three months for characterizing them and determining whether there was an outbreak. In this regard, the first polymyxin-resistant $A$. baumannii was identified in August 2016, and by the end of October 2016, the other five polymyxin-resistant $A$. baumannii isolates were identified. Our 


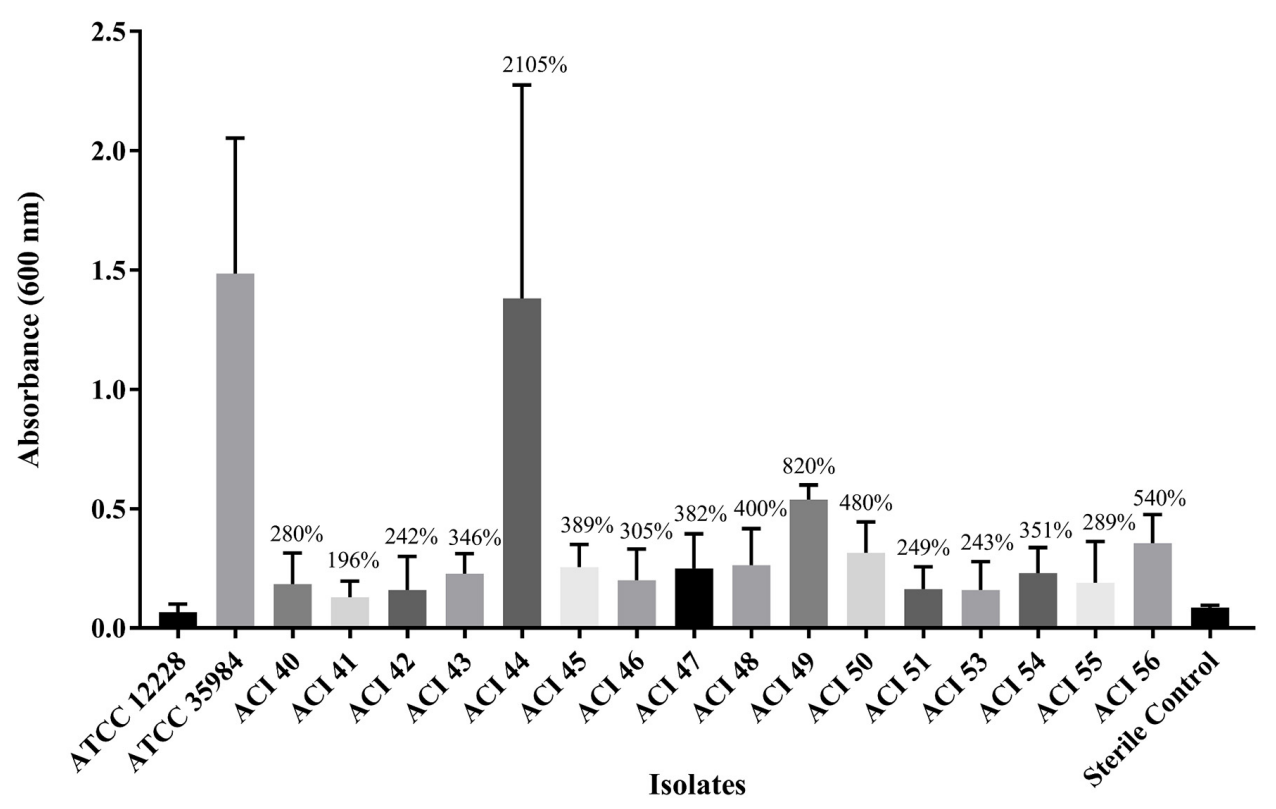

FIGURE 2 | Comparison of biofilm mass formed by A. baumannii strains (ACI) to that of Staphylococcus epidermidis ATCC 12228 and ATCC 35984 , the non-biofilm and the good biofilm former strains, respectively.

results showed that all isolates have $b l a_{O X A-51-l i k e}$ gene, thus belonging to the A. baumannii species. This species prevalence was expected and agreed with other studies in Brazilian hospitals (Leite et al., 2016; Castilho et al., 2017; Vasconcellos et al., 2017).

Regarding the antimicrobial susceptibility profile obtained (Figure 1), we noticed that it is quite common that the A. baumannii strains isolated in this hospital are XDR. In addition, if taken the reality at the local therapeutic protocol and all the antimicrobial options available to their patients into account, several isolates were classified by the hospital as ${ }_{\mathrm{L}}$ PDR because $25 \%$ of them were non-susceptible to all the antimicrobials available (Figure 1). Despite the reports that the A. baumannii strains isolated in Brazilian hospitals are MDR, the susceptibility of those isolates to the last choice drugs, such as polymyxins, still prevails (Leite et al., 2016; Sader et al., 2016; Castilho et al., 2017; Vasconcellos et al., 2017). For instance, in a Brazilian teaching hospital, Tavares et al. (2019) found MDR and XDR A. baumannii clones were emerging, including CC1 strains, as the second most prevalent; on the other hand, although MDR or XDR, none of the strains was resistant to polymyxin B (Tavares et al., 2019). Nevertheless, the high incidence of polymyxin resistance found in the A. baumannii isolates evaluated in this study $(37.5 \%)$ highlights the need for constant surveillance of antimicrobials resistance, particularly to the drugs used as last resources, in all the species isolated at the hospital. The high incidence of elevated tigecycline MIC found in the strains evaluated in this study (94\%) agrees with recent studies that analyzed Brazilian clinical isolates of A. baumannii (Cardoso et al., 2016; Vasconcellos et al., 2017), although historically A. baumannii Brazilian isolates are mostly susceptible to tigecycline (Leite et al., 2016; Sader et al., 2016; Castilho et al., 2017). The use of tigecycline as a treatment for
A. baumannii MDR strains infections is still controversial or, at least, not recommended, both in monotherapy or combined therapy (Ni et al., 2016; Eucast, 2017).

Being colonized by MDR A. baumannii is a risk factor for being infected by this microorganism (Fan et al., 2019). Skin decolonization with chlorhexidine bathing may help prevent catheter colonization and catheter-related bloodstream infections (Fan et al., 2019). Chlorhexidine MICs of all of our isolates are in a range already described in the literature for A. baumannii (Biswas et al., 2019; Nor A'shimi et al., 2019) with no difference among polymyxin-resistant and polymyxin-susceptible strains. The MICs (16-32 mg/L) were lower than the patient bathing solutions concentration ( $2 \%$ chlorhexidine gluconate, or $20,000 \mathrm{mg} / \mathrm{L}$ ), suggesting that the bathing would benefit the spread control if those strains colonized the patients' skin. Patient bathing with chlorhexidine and ventilators decontamination improvement (use of tubes with valves, ventilators not circulating to other patients until hospital discharge, plus being sterilized) were performed simultaneously as control measurements, and this way, the healthcare workers were able to control the dissemination of the polymyxin-resistant A. baumannii strains.

The biofilm is an important virulence factor that can ease the persistence of the bacterial cells in the environment and the host. In the biofilm form, they can evade immune responses and resist antibiotics more than in planktonic form (McConnell et al., 2013). The relationship between biofilmforming ability and antibiotic resistance is of great interest to clinicians and has already been established for Pseudomonas aeruginosa, for instance (Abidi et al., 2013), but remains unclear for A. baumannii (Qi et al., 2016). We wanted to verify whether a difference in biofilm formation among polymyxin-resistant and polymyxin-susceptible isolates existed. Our results show that 
all strains form biofilm at similar levels, but the polymyxinsusceptible strain ACI44 was the only one that formed more biofilm (Figure 2). It is not surprising to find in the literature that non-MDR A. baumannii isolates can form quantitatively more biofilm and more robust than MDR or XDR isolates. These resistant isolates usually are non-biofilm formers or tend to form weaker biofilms (Rodríguez-Baño et al., 2008; Qi et al., 2016). Thus, it seems that $A$. baumannii biofilms could be a mechanism for bacteria to get a better survival in the environment, especially when their resistance to antibiotics is not high enough (Qi et al., 2016).

The PFGE results showed a clonal similarity among five out of the six A. baumannii strains resistant to polymyxins in this hospital. The first isolate of the studied period (ACI51, subtype $\mathrm{B} 1$ ) is only possibly related to the other isolates resistant to polymyxins by PFGE (Figure 1). One should notice that the antimicrobial susceptibility profile of these strains belonging to the subtypes A2 and B1 are practically identical, which shows that antibiogram is not enough to discriminate the similarity of the strains, requiring molecular methodologies, such as PFGE, to distinguish different genotypic profiles of strains with similar phenotypes and define the occurrence of a possible outbreak. The patient's history from whom ACI51 was isolated shows recent hospitalization in another hospital, getting worst after five days discharged and then was admitted to the Teaching Hospital, where A. baumannii ACI51 was isolated from blood. Therefore, we suggest this pulsotype B ST79 strain is unrelated to the hospital endemic clone (pulsotype A).

The analysis of the draft genome sequence of five out of six polymyxin-resistant $A$. baumannii, plus two susceptible strains, shed light on the polymyxin-resistance and other mechanisms involved in the strains of this study.

ACI53 presented the higher tigecycline MIC and harbored an ORF codifying a major facilitator superfamily (MFS) antibiotic efflux pump, which was identified as tet(D)-like by CARD. Other mutations that may confer resistance to tigecycline, besides aminoglycosides, tetracyclines, fluoroquinolones, and chloramphenicol, are those in AdeSR, controlling the AdeABC efflux system expression level (Yilmaz et al., 2020). Substitutions in AdeRS were also already reported in colistin-resistant isolates. The draft-genomes of this study showed that the adeS gene of ACI54 and ACI55 presented a mutation leading to the Ala14Thr substitution; these strains were among those with the lowest tigecycline MIC.

Although there was a recent report of $m c r-4.3$ gene detection in an A. baumannii ST79 in Brazil (Martins-Sorenson et al., 2020), in the present study, the draft genome sequences excluded the $\mathrm{mor}$ genes in the emergence of polymyxin resistance among these isolates. Indeed, A. baumannii rarely harbors the $\mathrm{mcr}$ genes (Bitar et al., 2019; Hameed et al., 2019; Ma et al., 2019). The most common mechanisms of polymyxin resistance are usually related to chromosomal genes, such as those resulting in the loss of lipopolysaccharide by inactivation or insertion of sequence ISAba11 in the $l p x A, \operatorname{lp} x C$, or $l p x D$ genes (Moffatt et al., 2010, 2011; Henry et al., 2012) or due to the addition of phosphoethanolamine to the hepta-acylated lipid A by PmrC, which is regulated by $\mathrm{PmrAB}$, thus reducing the negative charge of the bacterial cells and consecutively reducing the affinity of polymyxins to their action target (Adams et al., 2009; Beceiro et al., 2011; Gerson et al., 2020).

Amino acid substitutions in the PmrCAB are known to confer colistin resistance (Lesho et al., 2013; Durante-Mangoni et al., 2015; Moffatt et al., 2019; Gerson et al., 2020) and are likely the only reason for the polymyxin resistance in this study's strains. We observed the substitution Asp82Asn in ACI51 PmrA, and although it is in the signal receiver domain, there is no mention in the literature relating this substitution to polymyxin resistance so far. Also, no phenotypic change was observed with Asp82Asn substitution when compared to the other strains. We subjected the mutated sequence to the SIFT algorithm ${ }^{6}$, which showed that the Asp82Asn substitution appears deleterious to protein activity.

Pro170Leu substitution in PmrB is known to cause an impact on the polymyxin resistance. In addition to Pro170Leu, Pro170Ser and Pro170Gln also increase $p m r C$ expression in polymyxin-resistant isolates (Arroyo et al., 2011; Gerson et al., 2020). The PmrB substitution Pro170Leu was present in ACI50 and ACI53. Curiously, these strains had higher polymyxin B MICs (>128 mg/L) when compared to the ACI51, ACI54, and ACI55 (from 16 to $64 \mathrm{mg} / \mathrm{L}$ ), which had a Thr232Ile substitution in PmrB, instead. ACI56 strain was indistinguishable to ACI50 and ACI53 by PFGE, belonging to subtype A2, and presented higher polymyxin B MIC (>128 mg/L) as well. For this reason, we analyzed ACI56 pmrB gene by PCR, revealing the same mutation found in ACI50 and ACI53. Colistin MICs of these strains were all at the same level ( $\geq 128 \mathrm{mg} / \mathrm{L})$. Thus, it appears that Thr232Ile substitution in PmrB may lead to a lower polymyxin B MIC than isolates with Pro170Leu substitution. The polymyxin B MICs in strains with Thr232Ile substitution in PmrB in strains from another study ranged from 8 to $96 \mathrm{mg} / \mathrm{L}$ (Lesho et al., 2013). According to the SIFT algorithm, although both substitutions are deleterious, the probability of Thr232Ile substitution (probability of substitution $=0.02$ ) is higher than the probability of Pro170Leu (probability of substitution $=0.00$ ). Therefore, the impact of the PmrB Thr232Ile substitution in polymyxin B activity needs further investigation.

All the resistant isolates had a mutation in $p m r C$, leading to the Arg125His replacement in PmrC. According to the SIFT algorithm, the Arg125His substitution appears to be tolerant to protein activity (probability of this substitution $=0.37$ ). Amino acid substitution in this position was already described, such as Arg125Pro, which has less impact on colistin resistance than amino acid changes in PmrA or PmrB (Gerson et al., 2020).

Because ACI40 (polymyxin-susceptible) was isolated 15 days apart after ACI50 (polymyxin-resistant), both from the same patient, the same lineage (ST1), and closely related to each other by PFGE, we concluded that different ST1 strains infected the patient during the hospital stay. Even so, we compared the genome of these strains and identified 30 mutated ORFs (Supplementary Table S2), of which eight were only present in ST1 polymyxin resistant isolates. Among them, an ORF that codifies acyl-CoA dehydrogenase gained our attention because of the involvement in the fatty acid breakdown and the membrane

\footnotetext{
${ }^{6}$ https://sift.bii.a-star.edu.sg/
} 
fluidity (Overath et al., 1969; Ma et al., 2015). Whether these mutations contribute to the mechanism of polymyxin resistance or are just related to the resistant clone expanding in the hospital, we still have to verify. In addition, the doubling-time shows that ACI40 presents a faster growth than ACI50, and some of these mutations likely affect the polymyxin-resistant isolate fitness (Pournaras et al., 2014). Further studies have to clear whether mutations leading to polymyxin-resistance simultaneously affect fitness.

Our results suggest that pulsotype A ST1 strains belong to an endemic clone that expanded in this hospital with recent mutations leading to polymyxin resistance. All the patients infected by the ${ }_{\mathrm{L}} \mathrm{PDR}$ strains received polymyxin B as treatment during the hospitalization period, contributing to the resistance selection. ACI51, the first polymyxin-resistant isolate identified in this hospital, belongs to ST79, and our results, along with the patient history, showed it is unrelated to the endemic clone.

A limitation of this study is that data on polymyxinresistant A. baumannii came from a single center. However, the main contribution of this study is the genomic evidence showing that carbapenem-resistant $A$. baumannii belonging to the international clone ST1 can easily expand in hospital settings and acquire chromosomal mutations in PmrB (Thr232Ile or Pro170Leu) and/or PmrC (Arg125His), resulting in the selection of polymyxin-resistant lineages displaying, at least, an ${ }_{\mathrm{L}}$ PDR phenotype.

In conclusion, we showed the clonal expansion of XDR A. baumannii ST1 strains in a Brazilian teaching hospital, including four ${ }_{\mathrm{L}} \mathrm{PDR}$ strains. The polymyxin resistance in this outbreak is due to chromosomal mutations, mainly with Pro170Leu substitution in PmrB. Further investigation is needed to know whether the Thr232Ile substitution in PmrB plays a role in the lower polymyxin B MICs. The transmission of these strains among patients points out the need for constant surveillance for adopting adequate measures to avoid new nosocomial outbreaks caused by XDR carbapenem-resistant $A$. baumannii strains.

\section{DATA AVAILABILITY STATEMENT}

The datasets presented in this study can be found in online repositories. The names of the repository/repositories and accession number(s) can be found in the article/ Supplementary Material.

\section{ETHICS STATEMENT}

The studies involving human participants were reviewed and approved by the Collegiate of the Teaching, Research,

\section{REFERENCES}

Abidi, S. H., Sherwani, S. K., Siddiqui, T. R., Bashir, A., and Kazmi, S. U. (2013). Drug resistance profile and biofilm forming potential of Pseudomonas aeruginosa isolated from contact lenses in Karachi-Pakistan. BMC Ophthalmol. 13:57. doi: 10.1186/1471-2415-13-57 and Extension Unit-NEPE/HRTN (NEPE 26/2017). Written informed consent for participation was not required for this study in accordance with the national legislation and the institutional requirements. The isolates of this study were registred at SISGEN under the number A2AAE3C.

\section{AUTHOR CONTRIBUTIONS}

LC conducted the susceptibility tests and wrote the manuscript. $\mathrm{AD}$ conducted the genome sequencing and analysis and wrote the manuscript. $\mathrm{CB}$ conducted the genome comparison and variant calling. GR conducted the biofilm assays and growth curves. IS conducted the analysis of chlorhexidine. JD conducted the MLST by PCR. CM responsible for organizing the data of the clinical lab from Vitek 2 and collaborate with suggestions to the manuscript. EL infectologist of the Hospital and leader of the microbiology lab, who organized the project for the sample storage and collaborated with discussions regarding the transmission in the hospital. CA responsible for isolating, identifying, and storing the bacterial collection in the clinical lab. NL conducted the genome sequencing and analysis and collaborate with suggestions to the manuscript. IC principal investigator of the group who organized the project, team, and results, and wrote part of the manuscript. All authors contributed to the article and approved the submitted version.

\section{FUNDING}

São Paulo Research Foundation (FAPESP 2013/07600-3) partially supported this study. Coordenação de Aperfeiçoamento de Pessoal de Nível Superior (CAPES no. 88882.328750/2019-01) supported CB. FAPESP, processes 2013/24952-0 and 2018/158874, supported $\mathrm{AD}$ and GR, respectively. PUB-USP, processes 2019/142 and 2017/705, supported IS and JD.

\section{SUPPLEMENTARY MATERIAL}

The Supplementary Material for this article can be found online at: https://www.frontiersin.org/articles/10.3389/fmicb. 2021.622704/full\#supplementary-material

Supplementary Figure 1 | Growth curves of $\mathrm{ACl} 40$ and $\mathrm{ACl} 50$. The data are from two independent experiments with six replicates for each strain.

Supplementary Table 1 | Genome assembly statistics and accession information.

Supplementary Table 2 | Mutations that were identified in the ACl50 when compared to $\mathrm{ACl} 40$.

Adams, M. D., Nickel, G. C., Bajaksouzian, S., Lavender, H., Murthy, A. R., Jacobs, M. R., et al. (2009). Resistance to colistin in Acinetobacter baumannii associated with mutations in the PmrAB two-component system. Antimicrob. Agents Chemother. 53, 3628-3634. doi: 10.1128/aac.00284-09

Arroyo, L. A., Herrera, C. M., Fernandez, L., Hankins, J. V., Trent, M. S., and Hancock, R. E. W. (2011). The pmrCAB operon mediates polymyxin 
resistance in Acinetobacter baumannii ATCC 17978 and clinical isolates through phosphoethanolamine modification of lipid A. Antimicrob. Agents Chemother. 55, 3743-3751. doi: 10.1128/aac.00256-11

Beceiro, A., Llobet, E., Aranda, J., Bengoechea, J. A., Doumith, M., Hornsey, M., et al. (2011). Phosphoethanolamine modification of lipid a in colistin-resistant variants of Acinetobacter baumannii mediated by the pmrAB two-component regulatory system. Antimicrob. Agents Chemother. 55, 3370-3379. doi: 10.1128/ aac.00079- 11

Biswas, D., Tiwari, M., and Tiwari, V. (2019). Molecular mechanism of antimicrobial activity of chlorhexidine against carbapenem-resistant Acinetobacter baumannii. PLoS One. 14:e0224107. doi: 10.1371/journal. pone. 0224107

Bitar, I., Medvecky, M., Gelbicova, T., Jakubu, V., Hrabak, J., Zemlickova, H., et al. (2019). Complete nucleotide sequences of mcr-4.3-carrying plasmids in Acinetobacter baumannii sequence type 345 of human and food origin from the czech republic, the first case in Europe. Antimicrob. Agents Chemother. 63:e01166-19.

Boucher, H. W., Talbot, G. H., Bradley, J. S., Edwards, J. E., Gilbert, D., Rice, L. B., et al. (2009). Bad bugs, no drugs: no ESKAPE! An update from the Infectious Diseases Society of America. Clin. Infect. Dis. 48, 1-12. doi: 10.1086/595011

Cai, Y., Chai, D., Wang, R., Liang, B., and Bai, N. (2012). Colistin resistance of Acinetobacter baumannii: clinical reports, mechanisms and antimicrobial strategies. J. Antimicrob. Chemother. 67, 1607-1615. doi: 10.1093/jac/dks084

Cardoso, J. P., Cayô, R., Girardello, R., and Gales, A. C. (2016). Diversity of mechanisms conferring resistance to $\beta$-lactams among OXA-23-producing Acinetobacter baumannii clones. Diagn. Microbiol. Infect. Dis. 85, 90-97. doi: 10.1016/j.diagmicrobio.2016.01.018

Castilho, S. R. A., Godoy, C. S. M., Guilarde, A. O., Cardoso, J. L., André, M. C. P., Junqueira-Kipnis, A. P., et al. (2017). Acinetobacter baumannii strains isolated from patients in intensive care units in Goiânia, Brazil: molecular and drug susceptibility profiles. PLoS One. 12:e0176790. doi: 10.1371/journal.pone. 0176790

Clinical and Laboratory Standards Institute. (2018). Performance Standards for Antimicrobial Susceptibility Testing: Twenty-eighth Informational Supplement M100-S28. Wayne, PA, USA: CLSI.

Diancourt, L., Passet, V., Verhoef, J., Grimont, P. A., and Brisse, S. (2005). Multilocus sequence typing of Klebsiella pneumoniae nosocomial isolates. J. Clin. Microbiol. 43, 4178-4182. doi: 10.1128/jcm.43.8.4178-4182.2005

Dias, V. C., Diniz, C. G., Peter, A. C., Bastos, A. N., Bastos, V. Q., Bastos, L. Q., et al. (2016). Epidemiological characteristics and antimicrobial susceptibility among carbapenem-resistant non-fermenting bacteria in Brazil. J. Infect. Dev. Ctries. 10, 544-553. doi: 10.3855/jidc.6640

Durante-Mangoni, E., and Zarrilli, R. (2011). Global spread of drug-resistant Acinetobacter baumannii: molecular epidemiology and management of antimicrobial resistance. Future Microbiol. 6, 407-422. doi: 10.2217/fmb.11.23

Durante-Mangoni, E., Del Franco, M., Andini, R., Bernardo, M., Giannouli, M., and Zarrilli, R. (2015). Emergence of colistin resistance without loss of fitness and virulence after prolonged colistin administration in a patient with extensively drug-resistant Acinetobacter baumannii. Diagn. Microbiol. Infect. Dis. 82, 222-226. doi: 10.1016/j.diagmicrobio.2015.03.013

Durmaz, R., Otlu, B., Koksal, F., Hosoglu, S., Ozturk, R., Ersoy, Y., et al. (2009). The optimization of a rapid pulsed-field gel electrophoresis protocol for the typing of Acinetobacter baumannii, Escherichia coli and Klebsiella spp. Jpn. J. Infect. Dis. 62, 372-377.

Eucast. (2017)The European Committee on Antimicrobial Susceptibility Testing. Breakpoint tables for interpretation of MICs and zone diameters. Version 7.1. https://www.eucast.org (accessed July, 2017).

Fan, C. Y., Lee, W. T., Hsu, T. C., Lee, C. H., Wang, S. P., Chen, W. S., et al. (2019). Effect of chlorhexidine bathing on colonization or infection with Acinetobacter baumannii: a systematic review and meta-analysis. J. Hosp. Infect. 103, 284-292. doi: 10.1016/j.jhin.2019.08.004

Fernandes, M. R., McCulloch, J. A., Vianello, M. A., Moura, Q., Pérez-Chaparro, P. J., Esposito, F., et al. (2016). first report of the globally disseminated incx4 plasmid carrying the mcr-1 gene in a colistin-resistant Escherichia coli sequence type 101 isolate from a human infection in Brazil. Antimicrob. Agents Chemother. 60, 6415-6417. doi: 10.1128/aac.01325-16

Gales, A. C., Castanheira, M., Jones, R. N., and Sader, H. S. (2012). Antimicrobial resistance among Gram-negative bacilli isolated from Latin America: results from SENTRY antimicrobial surveillance program (Latin America, 2008-2010). Diagn. Microbiol. Infect. Dis. 73, 354-360. doi: 10.1016/j.diagmicrobio.2012.04. 007

Gerson, S., Lucaßen, K., Wille, J., Nodari, C. S., Stefanik, D., Nowak, J., et al. (2020). Diversity of amino acid substitutions in PmrCAB associated with colistin resistance in clinical isolates of Acinetobacter baumannii. Int. J. Antimicrob. Agents. 55:105862. doi: 10.1016/j.ijantimicag.2019.105862

Gundi, V., Dijkshoorn, L., Burignat, S., Raoult, D., and La Scola, B. (2009). Validation of partial rpoB gene sequence analysis for the identification of clinically important and emerging acinetobacter species. Microbiology 155(Pt 7), 2333-2341. doi: 10.1099/mic.0.026054-0

Hameed, F., Khan, M. A., Muhammad, H., Sarwar, T., Bilal, H., and Rehman, T. U. (2019). Plasmid-mediated mcr-1 gene in Acinetobacter baumannii and Pseudomonas aeruginosa: first report from Pakistan. Rev. Soc. Bras. Med. Trop. 52:e20190237.

Henry, R., Vithanage, N., Harrison, P., Seemann, T., Coutts, S., Moffatt, J. H., et al. (2012). Colistin-resistant, lipopolysaccharide-deficient Acinetobacter baumannii responds to lipopolysaccharide loss through increased expression of genes involved in the synthesis and transport of lipoproteins, phospholipids, and poly- $\beta-1,6-\mathrm{N}$-acetylglucosamine. Antimicrob. Agents Chemother. 56, 59-69. doi: $10.1128 /$ aac.05191-11

Hood, M. I., Becker, K. W., Roux, C. M., Dunman, P. M., and Skaar, E. P. (2013). Genetic determinants of intrinsic colistin tolerance in Acinetobacter baumannii. Infect. Immun. 81, 542-551. doi: 10.1128/iai.00704-12

Karaiskos, I., and Giamarellou, H. (2014). Multidrug-resistant and extensively drug-resistant Gram-negative pathogens: current and emerging therapeutic approaches. Expert. Opin. Pharmacother. 15, 1351-1370. doi: 10.1517/ 14656566.2014.914172

Kluytmans, J. (2017). Plasmid-encoded colistin resistance: mcr-one, two, three and counting. Euro Surveill. 22:30588.

Leite, G. C., Oliveira, M. S., Perdigão-Neto, L. V., Rocha, C. K., Guimarães, T., Rizek, C., et al. (2016). Antimicrobial combinations against pan-resistant Acinetobacter baumannii isolates with different resistance mechanisms. PLoS One. 11:e0151270. doi: 10.1371/journal.pone.0151270

Lesho, E., Yoon, E. J., McGann, P., Snesrud, E., Kwak, Y., Milillo, M., et al. (2013). Emergence of colistin-resistance in extremely drug-resistant Acinetobacter baumannii containing a novel pmrCAB operon during colistin therapy of wound infections. J. Infect. Dis. 208, 1142-1151. doi: 10.1093/infdis/jit293

Lin, M. F., and Lan, C. Y. (2014). Antimicrobial resistance in Acinetobacter baumannii: from bench to bedside. World J. Clin. Cases 2, 787-814. doi: 10.12998/wjcc.v2.i12.787

Liu, L., Feng, Y., Zhang, X., McNally, A., and Zong, Z. (2017). New variant of mcr3 in an extensively drug-resistant Escherichia coli clinical isolate carrying mcr-1 and bla(NDM-5). Antimicrob. Agents Chemother. 61:e01757-17

Liu, Y. Y., Wang, Y., Walsh, T. R., Yi, L. X., Zhang, R., Spencer, J., et al. (2016). Emergence of plasmid-mediated colistin resistance mechanism MCR-1 in animals and human beings in China: a microbiological and molecular biological study. Lancet Infect. Dis. 16, 161-168. doi: 10.1016/s1473-3099(15)00424-7

Loewen, P. C., Alsaadi, Y., Fernando, D., and Kumar, A. (2014). Genome Sequence of an Extremely Drug-Resistant Clinical Isolate of Acinetobacter baumannii Strain AB030. Genome Announc. 2:e01035-14.

Ma, D. K., Li, Z., Lu, A. Y., Sun, F., Chen, S., Rothe, M., et al. (2015). AcylCoA dehydrogenase drives heat adaptation by sequestering fatty acids. Cell 161, 1152-1163. doi: 10.1016/j.cell.2015.04.026

Ma, F., Shen, C., Zheng, X., Liu, Y., Chen, H., Zhong, L., et al. (2019). Identification of a novel plasmid carrying mcr-4.3 in an Acinetobacter baumannii strain in China. Antimicrob. Agents Chemother. 63:e00133-19.

Magiorakos, A. P., Srinivasan, A., Carey, R. B., Carmeli, Y., Falagas, M. E., Giske, C. G., et al. (2012). Multidrug-resistant, extensively drug-resistant and pandrug-resistant bacteria: an international expert proposal for interim standard definitions for acquired resistance. Clin. Microbiol. Infect. 18, 268-281. doi: 10.1111/j.1469-0691.2011.03570.x

Martins-Sorenson, N., Snesrud, E., Xavier, D. E., Cacci, L. C., Iavarone, A. T., McGann, P., et al. (2020). A novel plasmid-encoded mcr-4.3 gene in a colistinresistant Acinetobacter baumannii clinical strain. J. Antimicrob. Chemother. 75, 60-64. doi: 10.1093/jac/dkz413

McConnell, M. J., Actis, L., and Pachón, J. (2013). Acinetobacter baumannii: human infections, factors contributing to pathogenesis and animal 
models. FEMS Microbiol. Rev. 37, 130-155. doi: 10.1111/j.1574-6976. 2012.00344.x

Moffatt, J. H., Harper, M., Adler, B., Nation, R. L., Li, J., and Boyce, J. D. (2011). Insertion sequence ISAba11 is involved in colistin resistance and loss of lipopolysaccharide in Acinetobacter baumannii. Antimicrob. Agents Chemother. 55, 3022-3024. doi: 10.1128/aac.01732-10

Moffatt, J. H., Harper, M., and Boyce, J. D. (2019). Mechanisms of polymyxin resistance. Adv. Exp. Med. Biol. 1145, 55-71. doi: 10.1007/978-3-030-16373$0 \_5$

Moffatt, J. H., Harper, M., Harrison, P., Hale, J. D., Vinogradov, E., Seemann, T., et al. (2010). Colistin resistance in Acinetobacter baumannii is mediated by complete loss of lipopolysaccharide production. Antimicrob. Agents Chemother. 54, 4971-4977. doi: 10.1128/aac.00834-10

Ni, W., Han, Y., Zhao, J., Wei, C., Cui, J., Wang, R., et al. (2016). Tigecycline treatment experience against multidrug-resistant Acinetobacter baumannii infections: a systematic review and meta-analysis. Int. J. Antimicrob. Agents 47, 107-116. doi: 10.1016/j.ijantimicag.2015.11.011

Nor A’shimi, M. H., Alattraqchi, A. G., Mohd Rani, F., Ni, A. R., Ismail, S., Abdullah, F. H., et al. (2019). Biocide susceptibilities and biofilm-forming capacities of Acinetobacter baumannii clinical isolates from Malaysia. J. Infect. Dev. Ctries. 13, 626-633. doi: 10.3855/jidc.11455

Overath, P., Pauli, G., and Schairer, H. U. (1969). Fatty acid degradation in Escherichia coli. an inducible acyl-CoA synthetase, the mapping of oldmutations, and the isolation of regulatory mutants. Eur. J. Biochem. 7, 559-574. doi: 10.1111/j.1432-1033.1969.tb19644.x

Palazzo, I. C., Rehder, A., and Darini, A. L. (2007). Quantitative disk diffusion as a convenient method for determining minimum inhibitory concentrations of oxacillin for staphylococci strains. J. Microbiol. Methods 71, 186-190. doi: 10.1016/j.mimet.2007.08.014

Park, S. Y., Si, H. J., Eom, J. S., and Lee, J. S. (2019). Survival of carbapenemresistant Acinetobacter baumannii bacteremia: colistin monotherapy versus colistin plus meropenem. J. Int. Med. Res. 47, 5977-5985. doi: 10.1177/ 0300060519879336

Peleg, A. Y., Seifert, H., and Paterson, D. L. (2008). Acinetobacter baumannii: emergence of a successful pathogen. Clin. Microbiol. Rev. 21, 538-582. doi: 10.1128/cmr.00058-07

Poirel, L., Jayol, A., and Nordmann, P. (2017). Polymyxins: antibacterial activity, susceptibility testing, and resistance mechanisms encoded by plasmids or chromosomes. Clin. Microbiol. Rev. 30, 557-596. doi: 10.1128/cmr.00064-16

Pournaras, S., Poulou, A., Dafopoulou, K., Chabane, Y. N., Kristo, I., Makris, D., et al. (2014). Growth retardation, reduced invasiveness, and impaired colistin-mediated cell death associated with colistin resistance development in Acinetobacter baumannii. Antimicrob. Agents Chemother. 58, 828-832. doi: 10.1128/aac.01439-13

Qi, L., Li, H., Zhang, C., Liang, B., Li, J., Wang, L., et al. (2016). Relationship between antibiotic resistance, biofilm formation, and biofilm-specific resistance in Acinetobacter baumannii. Front Microbiol. 7:483. doi: 10.3389/fmicb.2016. 00483

Qin, N., Tan, X., Jiao, Y., Liu, L., Zhao, W., Yang, S., et al. (2014). RNA-seq-based transcriptome analysis of methicillin-resistant Staphylococcus aureus biofilm inhibition by ursolic acid and resveratrol. Sci. Rep. 4:5467.

Rau, R. B., de Lima-Morales, D., Wink, P. L., Ribeiro, A. R., Martins, A. F., and Barth, A. L. (2018). Emergence of mcr-1 producing Salmonella enterica serovar typhimurium from retail meat: first detection in Brazil. Foodborne Pathog. Dis. 15, 58-59. doi: 10.1089/fpd.2017.2346

Rodríguez-Baño, J., Martí, S., Soto, S., Fernández-Cuenca, F., Cisneros, J. M., Pachón, J., et al. (2008). Biofilm formation in Acinetobacter baumannii: associated features and clinical implications. Clin. Microbiol. Infect. 14, 276278. doi: 10.1111/j.1469-0691.2007.01916.x

Rossi, F., Girardello, R., Cury, A. P., Di Gioia, T. S., Almeida, J. N. Jr., and Duarte, A. J. (2017). Emergence of colistin resistance in the largest university hospital complex of São Paulo, Brazil, over five years. Braz. J. Infect. Dis. 21, 98-101. doi: 10.1016/j.bjid.2016.09.011

Ruzin, A., Immermann, F. W., and Bradford, P. A. (2010). RT-PCR and statistical analyses of adeABC expression in clinical isolates of Acinetobacter calcoaceticus-Acinetobacter baumannii complex. Microb Drug Resist. 16, 87-89. doi: $10.1089 / \mathrm{mdr} .2009 .0131$

Sader, H. S., Castanheira, M., Farrell, D. J., Flamm, R. K., Mendes, R. E., and Jones, R. N. (2016). Tigecycline antimicrobial activity tested against clinical bacteria from Latin American medical centres: results from SENTRY antimicrobial surveillance program (2011-2014). Int. J. Antimicrob. Agents 48, 144-150. doi: 10.1016/j.ijantimicag.2016.04.021

Satlin, M. J., Lewis, J. S., Weinstein, M. P., Patel, J., Humphries, R. M., Kahlmeter, G., et al. (2020). Clinical and Laboratory Standards Institute (CLSI) and European Committee on Antimicrobial Susceptibility Testing (EUCAST) position statements on polymyxin B and colistin clinical breakpoints. Clin. Infect. Dis. 71, e523-e529.

Sohrabi, N., Farajnia, S., Akhi, M. T., Nahaei, M. R., Naghili, B., Peymani, A., et al. (2012). Prevalence of OXA-type $\beta$-lactamases among Acinetobacter baumannii isolates from Northwest of Iran. Microb. Drug Resist. 18, 385-389.

Tacconelli, E., Carrara, E., Savoldi, A., Harbarth, S., Mendelson, M., Monnet, D. L., et al. (2018). Discovery, research, and development of new antibiotics: the WHO priority list of antibiotic-resistant bacteria and tuberculosis. Lancet Infect. Dis. 18, 318-327.

Tavares, L., de Vasconcellos, F., de Sousa, W., Rocchetti, T., Mondelli, A., Ferreira, A., et al. (2019). Emergence and persistence of high-risk clones among MDR and XDR A. baumannii at a Brazilian Teaching Hospital. Front. Microbiol. 9:2898. doi: 10.3389/fmicb.2018.02898

Vasconcellos, F. M., Casas, M. R. T., Tavares, L. C. B., Garcia, D. O., and Camargo, C. H. (2017). In vitro activity of antimicrobial agents against multidrugand extensively drug-resistant Acinetobacter baumannii. J. Med. Microbiol. 66, 98-102. doi: 10.1099/jmm.0.000422

Xavier, B. B., Lammens, C., Ruhal, R., Kumar-Singh, S., Butaye, P., Goossens, H., et al. (2016). Identification of a novel plasmid-mediated colistin-resistance gene, mcr-2, in Escherichia coli, Belgium, June 2016. Euro Surveill. 21:27.

Yilmaz, Ş., Hasdemir, U., Aksu, B., Altınkanat Gelmez, G., and Söyletir, G. (2020). Alterations in AdeS and AdeR regulatory proteins in 1-(1naphthylmethyl)-piperazine responsive colistin resistance of Acinetobacter baumannii. J. Chemother. 1-8. doi: 10.1080/1120009X.2020.1735118 [Epub ahead of print].

Zhang, H. Z., Zhang, J. S., and Qiao, L. (2013). The Acinetobacter baumannii group: a systemic review. World J. Emerg. Med. 4, 169-174.

Conflict of Interest: The authors declare that the research was conducted in the absence of any commercial or financial relationships that could be construed as a potential conflict of interest.

Copyright (c) 2021 Carrasco, Dabul, Boralli, Righetto, Carvalho, Dornelas, Martins da Mata, Araújo, Leite, Lincopan and Camargo. This is an open-access article distributed under the terms of the Creative Commons Attribution License (CC BY). The use, distribution or reproduction in other forums is permitted, provided the original author(s) and the copyright owner(s) are credited and that the original publication in this journal is cited, in accordance with accepted academic practice. No use, distribution or reproduction is permitted which does not comply with these terms. 